\title{
Téoros
}

Revue de recherche en tourisme

\section{Le fleuve des régions}

\section{Alyne Groleau}

Volume 6, numéro 2, juillet 1987

Le Saint-Laurent magnétique

URI : https://id.erudit.org/iderudit/1080509ar

DOI : https://doi.org/10.7202/1080509ar

Aller au sommaire du numéro

\section{Éditeur(s)}

Université du Québec à Montréal

\section{ISSN}

0712-8657 (imprimé)

1923-2705 (numérique)

Découvrir la revue

\section{Citer cet article}

Groleau, A. (1987). Le fleuve des régions. Téoros, 6(2), 26-29.

https://doi.org/10.7202/1080509ar

Ce document est protégé par la loi sur le droit d'auteur. L'utilisation des services d'Érudit (y compris la reproduction) est assujettie à sa politique d'utilisation que vous pouvez consulter en ligne.

https://apropos.erudit.org/fr/usagers/politique-dutilisation/
Cet article est diffusé et préservé par Érudit.

Érudit est un consortium interuniversitaire sans but lucratif composé de l’Université de Montréal, l'Université Laval et l'Université du Québec à Montréal. Il a pour mission la promotion et la valorisation de la recherche. https://www.erudit.org/fr/ 


\title{
Le fleuve des régions
}

\author{
Par Alyne Groleau*
}

Nous avons voulu connaître l'importance accordée au fleuve dans les projets de développement touristique des municipalités régionales de comtés (MRC). Pour ce faire, nous avons dépouillé les schémas d'aménagement des $40 \mathrm{MRC}$ riveraines du SaintLaurent. Par souci d'homogénéité, nous avons exclu les villes de Montréal et de Québec ainsi que les MRC touchant le bassin de l'Outaouais, soient celles de DeuxMontagnes, de Laval, des Moulins et de Sainte-Thérèse-de-Blainville.

Comme les schémas d'aménagement doivent représenter les grandes orientations de développement régional, nous estimons que la présence ou l'absence du Saint-Laurent au sein de ces préoccupations ainsi que la manière de l'aborder dans les schémas révèlent de façon significative la perception qu"en ont les MRC et, conséquemment, la place qu'elles lui réservent dans leurs projets de développement.

Au début du siècle, le fleuve faisait principalement l'objet d'une exploitation touristique pour fins de villégiature et de croisières, dans les régions de Charlevoix et du Bas-Saint-Laurent. L'ouverture de nouvelles destinations, rendue possible par les developpements de la technologie, a grandement contribué au déclin de ces premiers foyers touristiques au Québec.

Par la suite, l'attrait touristique du fleuve semble avoir sombré dans l'oubli. Vers 1970, de nombreux projets ont périodiquement tenté de le remettre sur la carte, mais leur succès fut plutốt mitigé.... Pour n'en citer que quelques-uns: Un fleuve, un parc, La couleur fluviale, Une fenêtre sur le fleuve, Berges Neuves, et d'une manière plus récente, Le pare de l'archipel.

Bien que ces projets n'eussent pas nécessairement pour but de redonner une vocation touristique au fleuve, ils eurent le mérite de réaffirmer de temps à autre ce qui semble être la "difficile" reconnaissance de notre affiliation historique avec lui...

- Geographe, specialised en developpement touristique, Alyne Groleau pripare un projet de doctorat sur le Saint-Laurent.
S'il est généralement admis que les grands fleuves soient porteurs de civilisation, le Saint-Laurent a joué un rôle important dans la naissance et la survie de la nation, comme le dit si bien Jean-Claude Lasserre: Non seulement le peuplement s'est dendu le long des rivages, mais une vérilable civilisation fluviale s'est developpée, caracterisee par une organisation de l'espace à partir du flewve. (1)

Témoin du passé, le Saint-Laurent continue de façonner le présent par sa vocation commerciale et industrielle. Sa valeur culturelle constitue, quant à elle, une ressource touristique importante. Toutefois, comme le souligne la MRC de Charlevoix dans son schéma d'aménagement: $\bar{I}$ semble bien évideni que la simple présence de potentiels (touristiques), qu'ils solent naturels ou culrurels, ne peut suffire a attirer de nombreux investissemenis (...) dans la région. Ces potentiels ont donc besoin d'être connus et mis en valeur selon leurs caractéristiques propres. ${ }^{2}$

Dans les lignes qui suivent, nous allons donc vérifier si les potentiels naturels et culturels du fleuve sont reconnus et de quelle façon les MRC ont l'intention de les mettre en valeur.

\section{La mise en valeur récréo-touristique}

Presque toutes les MRC riveraines du Saint-Laurent ont décidé de l'associer à diverses formes de tourisme. Ainsi, dans l'ensemble, on peut degager trois grandes orientations pour sa mise en valeur récréotouristique:

\footnotetext{
- la préservation du patrimoine écologique et environnemental,

- le plein air.

- la villégiature.
}

\section{La préservation}

Toutes ces MRC ont mis dans leurs priorités des mesures de protection de l'environnement qui englobent nécessairement les zones littorales du fleuve. Cette préoceupation traduit l'intérêtt écologique qu'il sus=cite. Dans l'ensemble, elles ont identifié sur leurs portions de rives de nombreux habitats fauniques (milieux de fraie et de migra- tion saisonniêre, lieux de nidification et sites de haltes migratoires pour la sauvagine, etc.), et la flore particulière du littoral est incluse dans le patrimoine naturel du fleuve. Pour ces milieux fragiles, elles ont délimité des zones destinćes ầ la conservation stricte ${ }^{(3)}$, qui $n^{\prime} y$ autorisent aucune autre fonction, ou a la conservation modéréé(4), qui subordonne les activités humaines a la protection des sites. Ces affectations pourront prendre la forme de parcs ou de réserves écologiques régionaes, avec sentiers d'interprétation chez certaines ${ }^{(5)}$. Comme le souligne la MRC de Rivière-du-Loup, "la préservation de ces territoires met en valeur leur caractère à la fois unique et fragile"(6). Ces MRC invitent, pour ce faire, leurs municipalités à adopter des règlements en mesures de contrôle efficaces.

Mềme si toutes reconnaissent l'importance de préserver leur patrimoine écologique, il ne faut pas perdre de vue, comme plusieurs ${ }^{(7)}$ l'ont souligné, que: (...) les objectifs des schemas ne soni pas une fin en soi et leur accomplissement dépendra avant tout de la participation ef des initiatives des gens du milieu ainsi que des intervenants extérieurs à celui-ci. ${ }^{\left({ }^{3}\right)}$

Par ailleurs, $90 \%$ des MRC ont prévu des aménagements légers afin de préserver les sites et de mettre en valeur la beauté des paysages. Elles ont identifie, par exemple, les routes panoramiques, les percees visuelles ainsi que les sites d'intérêt esthétique. Pour souligner ces privilèges naturels, ces MRC ont l'intention de compléter leurs réseaux de haltes routières et d'installer des belvédères ou des tours sur les points d'observation panoramiques, en plus d'améliorer la signalisation des sites d'intérêt sur les routes en bordure du fleuve. Quelques-unes veulent inviter les automobilistes pressés à utiliser les voies rapides afin de permettre à qui veut apprécier le paysage de circuler en securité sur les routes panoramiques. Elles encouragent en général leurs municipalités à entreprendre des mesures de contrôle efficaces pour éviter la pollution visuelle sur leurs territoires (ex.: La Mitis), La MRC du Bas-Richelieu étudie les possibilités d'ouvrir une fenêttre sur le fleuve en récu- 


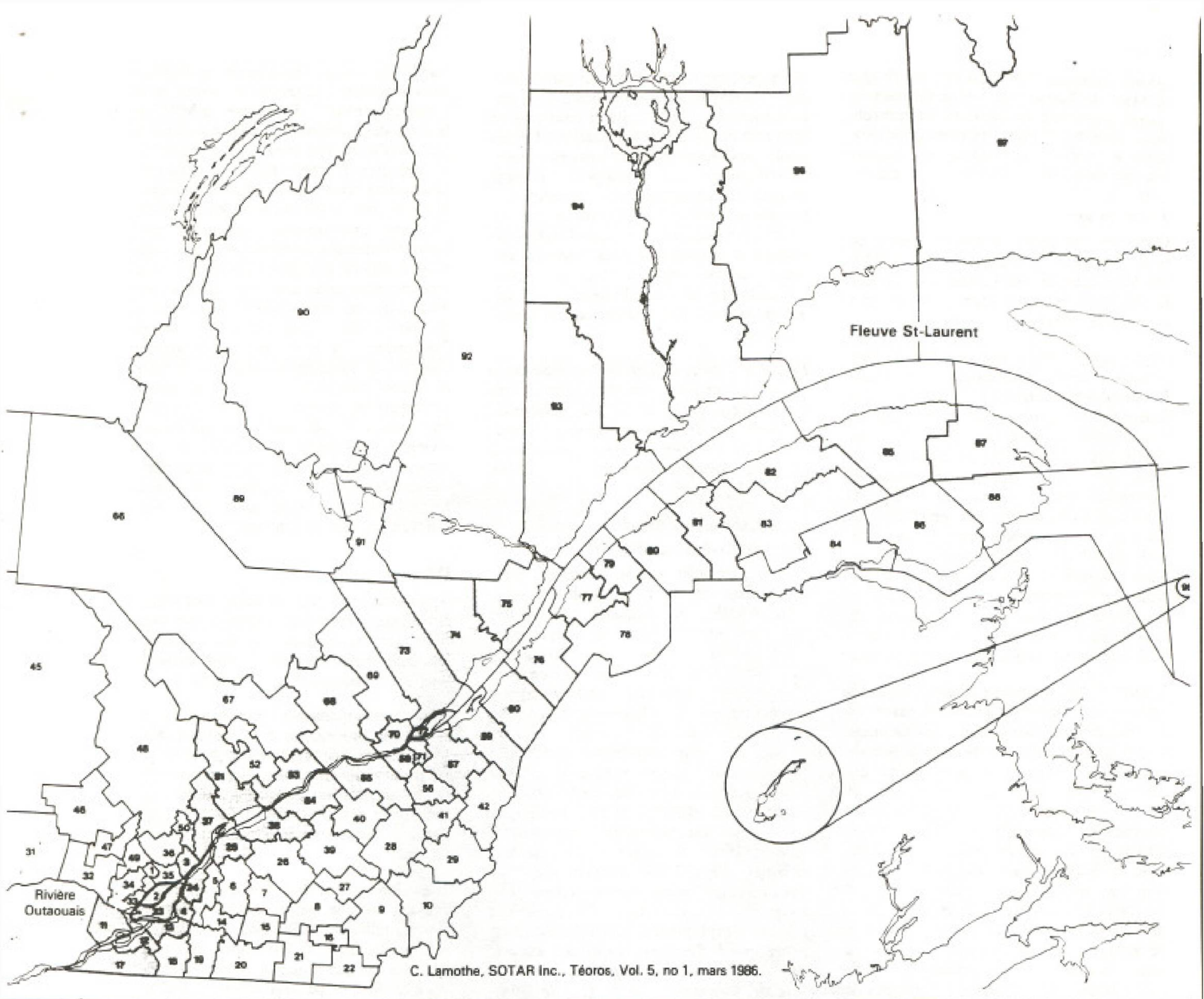

\section{Les MRC riveraines du Saint-Laurent}

Rive nord

97 Minganie

96 Sept-Rivières

94 Manicouagan

93 Haute-Côte-Nord

75 Charlevoix-Est

74 Charlevoix

73 Côte-de-Breaupré

72 L'lle d'Orléans

70 Communauté Urbaine de Québec*

68 Portneuf

53 Francheville

51 Maskinongé
37 D'Autray

3 L'Assomption

11 Vaudreuil-Soulanges

Rive sud

87 Côte-de-Gaspé

85 Denis-Riverin.

82 Matane

51 La-Mitis

80 Rimouski-Neigette

79 Les Basques

77 Rivière-du-Loup

76 Kamouraska

71 Desjardins

60 L'Islet
59 Montmagny

58 Chutes-de-la-Chaudière

57 Bellechasse

55 Lotbinière

54 Bécancur

38 Nicolet-Yamaska

25 Bas-Richelieu

24 Lajemmerais

4 Champlain

13 Roussillon

12 Beauharnois-Salaberry

17 Haut-Saint-Laurent

23 Communauté Urbaine de Montréal*

-Pour fin de localisation seulement 
pérant les zones d'inondation( ${ }^{(\%)}$, tandis que la MRC de Roussillon "incite les municipalités a adopter des mesures de contrôle permettant de dégager les rives et de protéger le potentiel ecologique, en laissant I'usage privé des rives de façon exceptionnelle" "(10์)

\section{Le plein air}

Outre des affectations de préservation et de mise en valeur écologiques, plus de $72 \%$ des MRC étudiées ont 1 'intention d utiliser le fleuve et ses rives pour en développer le potentiel récréo-touristique.

Profitant de sa proximite, les MRC de Bellechasse, Roussillon, Bas-Richelieu et Les Basques entre autres, ont l'intention d'y aménager des pistes cyclables, à même les voies ferrées désaffectées. Plusieurs veulent mettre en valeur leur localisation riveraine par l'aménagement de parcs et d'espaces verts. Ainsi, la MRC de Beauharnois-Salaberry veut créer un parc riverain, celle de Bellechasse un parc fluvial, la MRC L'Assomption un pare du Saint-Laurent alors que celle de Montmagny songe à aménager un parc national historique à Grosse-Ile, etc. Ces projets s'ajoutent à ceux de création des parcs régionaux et urbains en bordure du fleuve.

D'autres MRC projettent de développer les activités nautiques sur le Saint-Laurent ou à 1 'intérieur des baies et sur les battures. Celles dé la Haute-Cóte-Nord et la Minganie, en aval de Tadoussac, se proposent d'y développer la croisière, les excursions de peche et d'observation et la cueillette de coquillages. Les MRC de Charlevoix et Charlevoix-Est, de Portneuf, de Francheville, de Nicolet-Yamaska, de L'Assomption, de Roussillon, de Champlain, de Montmagny, de L'Islet, de Bellechasse et de Rivière-du-Loup veulent pour leur part développer la voile, la planche à voile, le canot, et aménager des plages, des marinas, des ports de refuges et des rampes de mise à l'eau. Elles prévoient aussi la réfection des quais et le nettoyage des berges, l'aménagement d'aires de repos et de piquenique avec stationnement et services usuels.

\section{La villegiature}

Les rives du fleuve sont très recherchées pour la beauté exceptionnelle du paysage. En raison de la popularité croissante de la villégiature, $75 \%$ des MRC ont prévu des zones à cette fin; pour le respect et la sauvegarde de la qualité visuelle, elles préconisent deux modèles d'organisation, le regroupement contrôlé des résidences secondaires et l'implantation à faible densité dans des secteurs soumis à des normes de protection. La villégiature a déjà pris un essor considérable dans plusieurs régions. Devant le fait accompli, les MRC incitent les municipalités à prendre les mesures adéquates pour empecher la dégradation visuelle des sites et y resserrer le contrôle du lotissement, de la construction et de l'architecture.
Cet aspect mérite une attention particulière car il souleve la question délicate de la privatisation des rives. Celle-ci constitue en effet l'un des principaux obstacles à l'accès public aux berges et à leur mise en valeur. La villégiature étant plutồt perçue comme un luxe, elle accentue le grignotement d'un patrimoine collectif déjà entamé par les "maux nécessaires" que sont l'industria. lisation, l'urbanisation et la commercialisation de ces espaces. On peut donc $s$ 'attendre à ce que la villégiature soit l"une des premières cibles touchéc par les enjeux régionaux...

Même s'il peut couler encore beaucoup d'eau sous les ponts, les MRC considèrent que de façon générale: (...) les actions de protection et de mise en valeur (...) peuvent creer des chäteaux-forts, destinés à certains groupes privilegies et bien nantis: (11)

\section{La mise en valeur du potentiel culturel}

Très peu de MRC ont su mettre en relief le röle historique du fleuve. Seulement $20 \%$ d'entre elles ont souligné son apport historico-culturel à leurs attraits patrimoniaux; parmi celles-ci, on retrouve les MRC de Matane, de Rivière-du-Loup, de Portneuf, D'Autray, qui ont su exprimer très clairement leur relation historique avec le Saint-Laurent. Par exemple, celle de Rivière-du-Loup manifeste nettement $1^{\prime}$ intention de valoriser la présence du fleuve et de mettre en évidence le caractère marin de la MRC ${ }^{(2)}$. Portneuf souligne que le fleuve est traditionnellement integré au mode de vie des gens (...), qu'il devient l'élément-cléf du développement économique de la MRC et que toute la richesse historique en témoigne bien... (13). Matane et D'Autray expliquent ainsi leur localisation: Attirés par le fleuve qui constituait à la fois une source de revenu (la péche) et la seule voie de communication ${ }^{(1.4)}$, (...) le SaintLaurent devint rapidement un axe de fixation des hommes et de leurs activités ${ }^{(15)}$.

Cependant, pour la majorité des MRC, la valeur culturelle du Saint-Laurent est abordée de manière moins explicite. Par exemple, elles établissent la localisation de nombreux sites archéologiques et patrimoniaux en bordure du fleuve, sans toutefois faire allusion ou référence aux rapports possibles entre le Saint-Laurent et cette localisation spécifique... Certaines ne font nullement allusion au fleuve et situent plutôt l'axe patrimonial en fonction de la route. Dans ces conditions, il faut savoir lesquels de ces routes ou ces sites patrimoniaux sont situes en bordure du fleuve...

D'autres MRC ne semblent pas avoir l'intention de profiter outre mesure des privilèges du fleuve. Par exemple, celle de l'lle-d'Orléans veut miser davantage sur le caractère symbolique des valeurs et des traditions rattachés au territoire québé- cois ${ }^{(t)}$, le fleuve y est abordé au chapitre des contraintes, en termes de pollution et d'usages perdus $^{(17)}$. De mème, la MRC de la Cóte-de-Beaupré, qui s'inscrit dans la plus vieille région rurale du Canadai18), $n$ 'y fait pas référence parmi les élèments. de son riche patrimoine; le fleuve n'y figure ni en termes de localisation pour certains ćléments patrimoniaux, ni en termes d'attrait esthétique ou touristique. Les póles touristiques de la région, qui ont déjà une certaine renommée, gravitent plutót autour des chutes, des parcs pour la montagne et la forét et l'observation des oiseaux au cap Tourmente. La MRC de Sept-Rivières, quant à elle, a l'intention de mettre en place un circuit régional axé (...) sur la culture amérindienne, la pêche, la forềt et l'industrie minière ${ }^{19}$; elle mise davantage sur ses rivières sans accorder trop d'importance au fleuve, bien qu'elle prévoit un parc régional des Sept-Iles qui saura peut-étre mettre en valeur malgré tout les divers. potentiels du Saint-Laurent.

\section{Bilan}

Apparemment, toutes les MRC sont préoccupées par la protection du patrimoine naturel, non seulement en fonction du développement touristique mais aussi de tous leurs champs d'interventions.

Elles semblent attacher beaucoup d'importance à la sauvegarde et a la valorisation de la beauté des paysages, et insistent sur les mesures de contróle à adopter pour éviter la dégradation du paysage. Elles veulent, entre autres, souligner les privilèges naturels des sites en améliorant la signalisation.

Il est intéressant de noter que des MRC n'ayant qu'une petite ouverture sur le fleuve, telles les MRC de L'Assomption, du Bas-Richelieu, de Roussillon, de Champlain, ainsi que d'autres situées dans des régions dites de passage comme La Mitis, Les Basques, Portneuf, aient l'intention de mettre en valeur les attraits écologiques et récréo-touristiques du fleuve sur leur territoire.

Ces projets constituent de réels progrès en matière de reconnaissance ec d'uctilisation de cette grande ressource qu'est le SaintLaurent qui a trop longtemps servi de dépotoir et d'égout a ciel ouvert... Mais il reste beaucoup à faire pour le dépolluer et permettre de manière satisfaisante l'utilisation récréative de ses eaux. Par leurs différents projets de protection et de mise en valeur, les MRC se préparent à prendre des mesures rigoureuses pour corriger la situation.

Par contre, les potentiels culturels du SaintLaurent sont en général méconnus et sousdéveloppés. La majorité des MRC localisent en bordure du fleuve ou de la route les sites patrimoniaux les plus anciens mais ne précisent pas que ces routes longent le Saint-Laurent et qu'il a contribué à l'éta- 
blissement et à la survie des ancëtres. Un peu comme si notre discours culturel, principalement axé sur la colonisation, niait ses assises historiques et géographiques.

Bien entendu, il nous faut rappeler que cette étude ne rend pas compte des études commandées en dehors des schemas, pour la mise en valeur du fleuve ${ }^{1201}$. Précisons en outre que quelques-unes des MRC n'en étaient qu'à l'étape préliminaire des propositions d'aménagement. S'il n'est pas exclu que cette situation puisse évoluer en faveur d'une meilleure reconnaissance des potentiels culturels du Saint-Laurent, on peut espérer qu'elle contribue néanmoins à identifier les lacunes à corriger.

Dans l'actuel contexte de développement touristique régional, les MRC riveraines au fleuve font des efforts pour mettre en valeur leurs caractères distinctifs. Cependant, devons-nous souligner que ce fleuve qu"elles ont en commun les differencie les unes des autres, tant par la diversité de son paysage que par sa contribution historique à l'enracinement humain et à la survie de chacune... Et que ce grand fleuve dont elles ont le privilège leur confere un caractère original qui les distingue depuis déjà longtemps... à travers le monde. Elles ont donc tout intérêt à le reconnaitre...

\section{Notes ot références}

(1) Lasserre, J.-C., Le Saint-Laurent grande porte de l'Amerique, Ed. Hurtubise HMH. Cahiers du Québec, Coll. Géographie, 1960, p. 91

(2) MRC de Charlevoix. Schema d'amenagament, p. 59 .

(3) C'est le cas des MAC de Champlain (D. 25), en Denis-Riverain $|\mathrm{D}, 30|$

(4) Par exemple dans le cas de la MAC de Matane. p. 52.

(5) Telles les MAC de Champlain ip, 11k, de L'Assomption (D. 62), de Francheville ID. 62).

(6) MRC de Riviere-du-Loup, Schéma d'amenage ment, p. 24

(7) Entre autres, les MRC de Champlain (p. 57) et de Poussillon (p. 32).

(8) MAC de Charievoix, Schéma d'amenagement. p. 2

(9) MAC du Bas-Richeliev, Schema d'amenagement, p. 35 .

(10) MAC de Roussillon, Schéma d'amenagement, p. 32

(11) MAC de Charlevoix, Schema d'aménagement, p. 21.

(12) MRC de Riviente-du-Loup, Schema d'amenage ment, pp. 11 et 54 .

(13) MAC de Portneuf, Sehema d'amánagement, $p$. 107 .

114) MRC de Matane, Schéma d'áménagement, p. 19

(15) MRC D'Autray, Schéma d'aménagement, p. 2
(16) MRC lle d'Oriteans, Proposition d'amenage ment, p. 149 ,

(17) Idem, p. 92

(18) MRC de la Cote-de-Buauné, Schema d aména. gement, p. 5 .

(19) MRC der Sept-Fivieres, Schéma d'amanago. ment. D. 22

(20) Comme c'est lie cas, entre autres, pour les MAC de Beauharnois-Salaberry et de Parneuf.

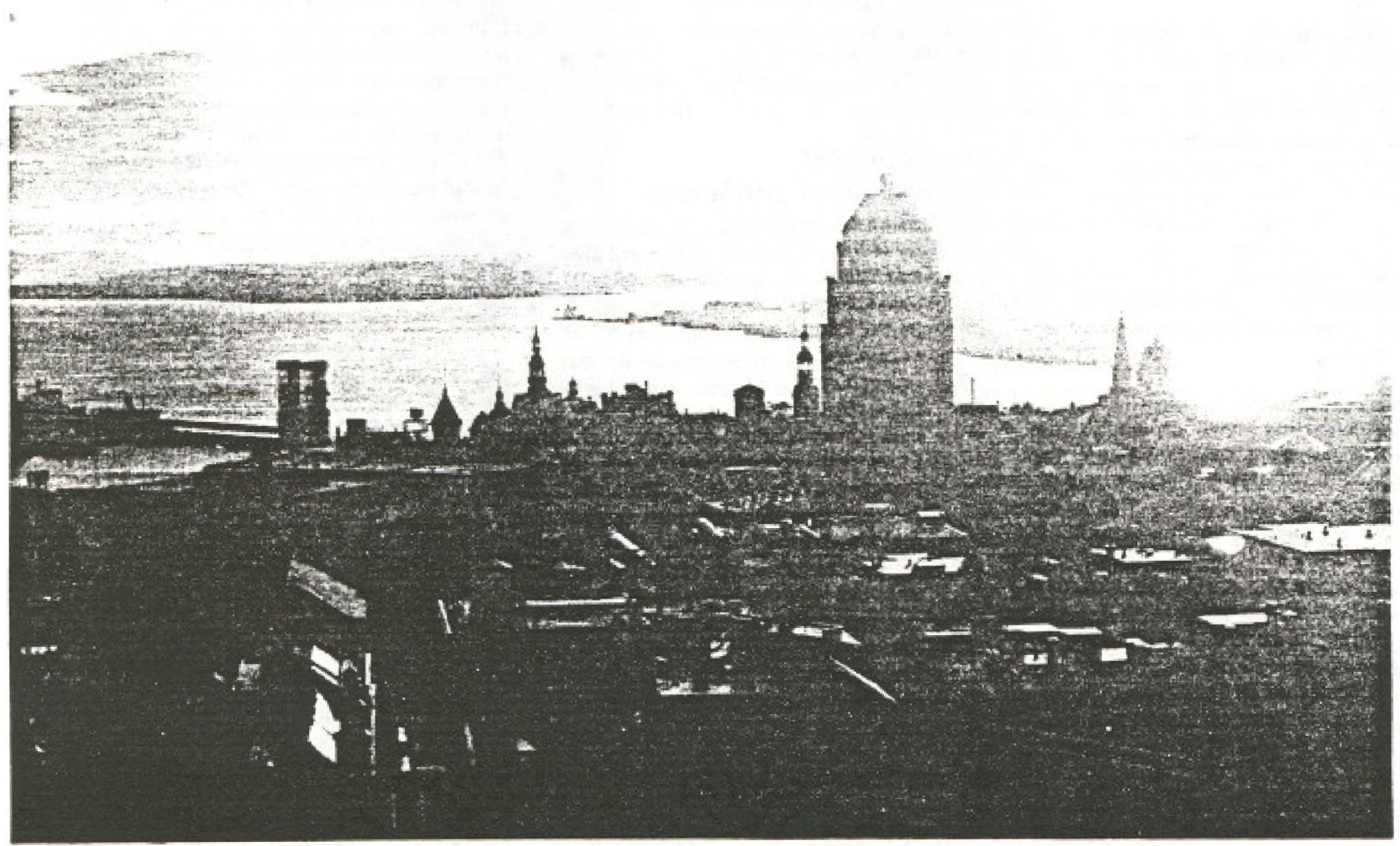

Le Saint-Laurent: une ressource id mottre en valeur. 